\title{
Pseudonocardia carboxydivorans in human cerebrospinal fluid: a case report in a patient with traumatic brain injury
}

\author{
Amalia Navarro-Martínez ${ }^{1 *}$ (D), Noelia Corominas ${ }^{1}$, Caridad Sainz de Baranda², Ángel Escudero-Jiménez², \\ Jorge Galán- Ros ${ }^{2}$, Juan Antonio Sáez- Nieto ${ }^{3}$ and Javier Solera ${ }^{1}$
}

\begin{abstract}
Background: Members of the genus Pseudonocardia have been widely reported and recovered from several ecosystems, such as soil samples and plant samples. Pseudonocardia bacteria colonize the microbial communities on the integument of fungus gardening ant species. We present the first documented case of Pseudonocardia carboxydivorans isolated in human cerebrospinal fluid (CSF). To the best of our knowledge, this is the first report of an human infection by $P$. carboxydivorans.
\end{abstract}

Case presentation: A patient, who suffered a traumatic brain injury a month before, was admitted to this hospital due to gait alteration and cognitive disturbances. Culture of cerebrospinal fluid showed ramified, not acid-fast, Gram positive bacilli. The bacterium was identified by molecular methods as P. carboxydivorans.

Conclusion: This is the first documented case of isolating $P$. carboxydivorans in human CSF in a case of probable meningitis. Further research is needed in order to determine its pathogenic role in human infections.

Keywords: Pseudonocardia carboxydivorans, Human cerebrospinal fluid (CSF), Traumatic brain injury (TBI), Carboxydobacteria

\section{Background}

Pseudonocardia carboxydivorans is a Carboxydobacteria. Carboxydobacteria are a group of aerobic bacteria that can grow aerobically on $\mathrm{CO}$ as the sole source of carbon and energy [1]. A bacterial strain, $\mathrm{Y}^{\mathrm{T}}$, capable of oxidizing carbon monoxide, was isolated from a soil sample collected from a roadside in Seoul, Korea. Strain $\mathrm{Y}^{\mathrm{T}}$ containes MK9 as the major menaquinone, which is different from major menaquinone reported previously in the genus Pseudonocardia, MK-8 $\left(\mathrm{H}_{4}\right)$. DNA-DNA relatedness between strain $\mathrm{Y}^{\mathrm{T}}$ and the type strains of $P$. alni and $P$. antarctica was respectively 10 and $63 \%$. Based on phylogenetic, morphological and chemotaxonomic evidence, it is proposed that strain $\mathrm{Y}^{\mathrm{T}}$ be classified as the type strain of a novel species, P. carboxydivorans sp.nov. [2].

\footnotetext{
* Correspondence: amalia.n.m@sescam.jccm.es

'Internal Medicine Department, University General Hospital, C/ Hermanos

Falcó s/n, 02006 Albacete, Spain

Full list of author information is available at the end of the article
}

Members of the genus Pseudonocardia have been widely reported and recovered from several ecosystems, such as soil samples and plant samples [2,3]. Pseudonocardia bacteria colonize the microbial communities on the integument of fungus-gardening ant species [4]. Pseudonocardia spp. is a known antifungal commensal microorganism [5] and a higer abundance of this taxon might rather reflect the presence of fungal organisms in the distal airways. The bronchoalveolar lavage fluid (BAL) microbiota of rheumatoid arthritis (RA) was significantly less diverse and abundant compared to healthy controls. The genus Pseudonocardia were the only taxa over represented in RA BAL and correlated with higher disease activity and erosions [6]. To the best of our knowledge, this is the first report of a probable human infection by P.carboxydivorans. That is the first documented case of isolating P.carboxydivorans in human CSF. 


\section{Case presentation}

A 67-year-old patient was admitted to this hospital due to gait alteration, cognitive disturbances and rapid clinical deterioration. The patient had a history of diabetes, asthma and a myocardial infarction 2 years earlier. Medications at the time of admission were acetylsalicylic acid, clopidogrel, glimepiride, pitavastatin and omeprazole. For the previous 2 years, he had intermittent syncope episodes. In one of them he suffered a fracture of the left tibia. Encephalogram, electrocardiogram (ECG), subcutaneos Holter, echocardiography and stress ECG were normal. A month before admission, hehad a new syncope episode while cultivating vegetables and he suffered a traumatic brain injury (TBI) with a left orbital and right occipital hematoma and he went to the Emergency Department of the Albacete General Hospital. A computed tomography (CT) showed a left subdural and retroorbital hematoma as well as a right occipital skull fracture. Neurologic examination was normal. A week after the injury, he suffered a respiratory infection with cough and yellow mucus and his primary care physician treated him with amoxicillin-clavulanic acid (875/125 mg every 8 h, 7 days, orally) with a consequent improvement of the respiratory symptoms. Then he was referred to the Internal Medicine Department for presenting continuous headache since the head injury and symptoms of progressive weakness in the lower limbs with instability while walking and confusion. The patient maintained normal vital signs and his body temperature was $36.5^{\circ} \mathrm{C}$. No skin lesions, lymph nodes or goiter were present. He carried a subcutaneous Holter device with no signs of local infection. Cardiopulmonary and abdominal explorations were normal. No edemas were present and the pulses were palpable. He showed disorientation to time and place, but not person. He had a paresis of the left seventh cranial nerve. He had a minimal decrease in strength in the lower limbs. Both the proprioceptive and the tactile sensitivity were diminished in the lower limbs, and showed no sensitivity to vibration. The tendinous reflexes were diminished in the examination. The finger-to-nose testing was impaired. The Romberg test was positive. The patient had gait disturbance with widened base and inability for tandem gait. He was admitted to the Internal Medicine Department. Laboratory tests were performed. Routine hematological testing showed a total hemoglobin $14.4 \mathrm{~g} / \mathrm{dl}$ and a normal leucocyte and platelet count.Erytrocyte sedimentation raye was $51 \mathrm{~mm} / 1 \mathrm{~h}$ (normal range values $1-20 \mathrm{~mm} / 1 \mathrm{~h}$ ). Prothrombin time and partial -thromboplastin time were normal. All blood chemistry values were normal except the values for glucose and C-reactive protein (CRP). The glucose concentration was $200 \mathrm{mg} / \mathrm{dl}$ (normal range values $74-109 \mathrm{mg} / \mathrm{dl}$ ), the CRP level was $11.8 \mathrm{mg} / \mathrm{l}$ (normal range values $0-5 \mathrm{mg} / \mathrm{l}$ ). Blood and urine cultures were negative. Chest $\mathrm{x}$-ray was normal.
Serology for Treponema pallidum, Coxiella burnetii, BrucellaBorrelia burgdorferi, HIV, Cytomegalovirus, Epstein Barr virus, Simple herpes virus, Toxoplasma, Mycoplasma pneumoniae was negative for acute infection with no seroconversion. A contrast magnetic resonance was carried out which revealed a right occipital fracture with a left frontobasal hematoma, a small left subdural occipital hematoma, a right occipital fracture with laminar subdural hematoma and a frontobasal contusion. A thoracoabdomino-pelvic CT was carried out with no pathological findings. An electromyography (EMG) showed a sensory and motor mixed polyneuropathy with a severe demyelinization component. Moreover the patient showed a diabetic peripheral polyneuropathy. A lumbar puncture was carried out and CSF was obtained with a cell count of 105 cells/mcl with $100 \%$ mononuclear cells, glucose $100 \mathrm{mg} / \mathrm{dl}$, $1.44 \mathrm{~g} / \mathrm{l}$ proteins. As for the microbiological study, Gram staining of acid-fast bacilli, as well as aerobic,anaerobic,fungi,Mycobacteria and Brucella culture,were negative. Due to a positive tuberculin skin test (purified protein derivative, 5 TU) of $28 \mathrm{~mm}$, a urine culture for Mycobacteria and a polymerase chain reaction (PCR) as well as a culture for Mycobacterium tuberculosis in CSF were requested and were negative. Faced with the possibility of a disimmune process and, after receiving the results of the electromyogram, it was decided on a treatment with $0.4 \mathrm{mg} / \mathrm{kg} /$ day intravenous immunoglobulin for 5 days with improvement of the clinical symptoms, recovery of the tendinous reflexes and the disappearance of the gait alteration. A second lumbar puncture was requested to check the characteristics of the fluid during treatment. The white blood cell count was 28 cells $/ \mathrm{mcl}$, predominantly mononuclear, no malignant cells, $0.88 \mathrm{~g} / \mathrm{l}$ protein and adenosine deaminase (ADA) $5.9 \mathrm{u} / \mathrm{l}$. The cytology of CSF showed lymphoplasmacytosis. In the microbiological analysis of CSF, Gram staining showed a few polymorphonuclear leukocytes and absence of microbial flora. The aerobic culture was performed on blood agar medium, chocolate agar medium and selective medium for Legionella (BCYE). After 7 days of incubation at $37{ }^{\circ} \mathrm{C}$ and $5 \% \mathrm{CO}_{2}$, the culture showed growth of white, dry and rough colonies (Fig. 1, Panel a), which were presumably identified as Actinomyces spp. by microbiological routine testing such as the Gram stain (Panel B) and Kinyoun stain (Panel C), showing the presence of ramified, not acidfast, Gram-positive rods.. The bacterium was identified by molecular methods of rRNA $16 \mathrm{~S}$ sequencing,as Pseudonocardia carboxydivorans in the National Center of Microbiology (ISCIII, Madrid, Spain). The isolate was identified by means of $16 \mathrm{~s}$ rRNA sequence analysis using a previously reported method [7] The $1434 \mathrm{bp}$ fragment obtained from the isolate showed a similarity of $99.7 \%$ with $P$. carboxydivorans (GeneBank accession numbers FJ547123, NR044092 and others). When using 


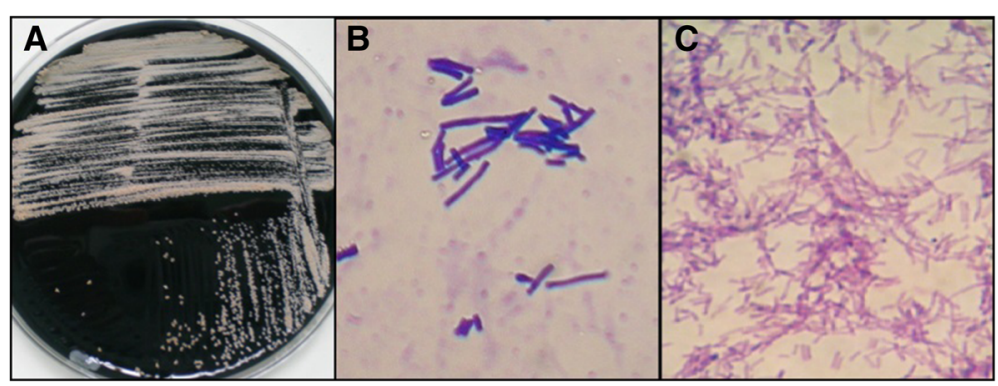

Fig. 1 Microbiologic outcomes in cerebral spinal fluid. Panel a shows the culture of the specimen obtained from SCF the patient on selective medium for Legionella (BCYE) showing growth of white, dry and rough colonies after 7 days of incubation at $37^{\circ} \mathrm{C}$ and $5 \% \mathrm{CO} 2$ atmosphere. Panels $\mathbf{b}$ and $\mathbf{c}$ show the microscopic examination of the colonies by Gram and Kinyoun modified strain, showing the presence of ramified, not acid-fast, Gram-positive bacilli. The bacterium was identified by molecular methods of RNAr 165 sequencing, being finally identified as Pseudonocardia carboxydivorans in the National Center of Microbiology (ISCIII, Madrid, Spain)

E -test, the strain was sensitive (MICs, $\mu \mathrm{g} / \mathrm{ml})$ to all antimicrobials tested: ciprofloxacin (0.12), amikacin (0.5), trimethoprim-sulfamethoxazole (2), linezolid (0.25), imipenem (0.12), amoxycilin-clavulanic (1), cefotaxime (0.5) and erythromycin (2) Treatment with trimethoprimsulfamethoxazole was initiated $(20 \mathrm{mg} / \mathrm{Kg}$ every $6 \mathrm{~h})$ intravenously. $\mathrm{He}$ did not receive any other antibiotic treatment added.Once this germ was isolated and to complete the study, a third lumbar puncture was carried out (Table 1). The CSF flow cytometry was normal. Besides, a biopsy of the bone marrow and a karyotype were conducted, which were normal, and a PET-CT showed the absence of metabolically significant localization at any level.

The patient received 3-week regimen of trimethoprimsulfamethoxazole $20 \mathrm{mg} / \mathrm{Kg}$ every $6 \mathrm{~h}$ intravenously followed by oral treatment with trimethoprimsulfamethoxazole $(800 / 160 \mathrm{mg}$ twice a day). Two and 10 weeks after initiating treatment, new CSF control samples were taken, where $P$. carboxydivorans was isolated again; however, the characteristics of the liquid were normal. The duration of antimicrobial therapy was 10 months. The patient declined a new lumbar puncture in the absence of symptoms. Patient follow-up at 15 months has shown a favorable evolution, with no relapse of symptoms.

\section{Discussion}

The genus Pseudonocardia was first established by Henssen in 1957 [1] and since then the genus description has been revised adding new species [2].

Pseudonocardia is a known antifungal commensal microorganism $[4,5]$ and a higher abundance of this taxon might rather reflect the presence of fungal organismsin the distal airways. A bibliographic search on Pubmed, Medline, Up to Date and other search platforms on pathologies caused by this germ rendered only microbiological description but no clinical cases in human. The genus Pseudonocardia were the only taxa over represented in RA BAL and correlated with higher disease activity and erosion [6] To the best of our knowledge, this is the first report of a probable human infection by P.carboxydivorans. That is the first documented case of isolating P.carboxydivorans in human CSF.

Patients with traumatic brain injury (TBI) are at a high risk for developing infections. Respiratory tract infections are the most common, with Acinetobacter spp. being the emerging pathogens. In a recent study, meningitis developed in $2 \%$ of the patients, whereas surgical site infections other than meningitis occurred in $4.25 \%$ [8]. Communication of the CSF with the environment was a major risk factor for the development of both SSI and meningitis.

Table 1 Profile of laboratory results among the 4 CFS samples collected from initial ${ }^{a}$

\begin{tabular}{llllllll}
\hline Sample no & $\begin{array}{l}\text { Date of sample collection } \\
\text { (day } / \mathrm{mo} / \mathrm{yr})\end{array}$ & Cell count cells/mcl & Cell type & ADA (u/l) & Proteins g/l & Glucose mg/dl & CSF culture result \\
\hline 1 & $24 / 05 / 2013$ & 105 & $100 \% \mathrm{MN}$ & $\mathrm{ND}$ & 1.44 & 90 & Neg (Pseudonocardia ND) \\
2 & $05 / 06 / 2013$ & 28 & $100 \% \mathrm{MN}$ & 5.9 & 0.88 & 84 & P. carboxydivorans \\
3 & $20 / 06 / 2013$ & 10 & $100 \% \mathrm{MN}$ & 5.8 & 0.78 & 96 & P. carboxydivorans \\
4 & $27 / 08 / 2013$ & 1 & 1 red blood cell & $\mathrm{ND}$ & 0.71 & 92 & P. carboxydivorans \\
\hline
\end{tabular}

${ }^{a} N D$ no data, Neg negative, $M N$ mononuclear 
The portal of entry of $P$. carboxydivorans in this patient remains speculative. Carboxydobacteria are a group of aerobic bacterias, which have been isolated in soil samples. The patient suffered a syncope episode while cultivating vegetables and he suffered a traumatic brain injury (TBI), but with no neurosurgical procedure or open fracture. Pseudonocardia could have developed in the soil he cultivated and get in contact with the patient in the traumatic brain injury. The respiratory tract infection might have been another gate of entrance, since Pseudonocardia is a well-known antifungal commensal and a higher abundance of this taxon might rather reflect the presence of fungal organism in the distal airways. But this patient not had any fungal infections, not breathing or rheumatic pathologies, so we though this way of entrance would be improbable.

Our patient suffered a TBI with a left orbital and right occipital hematoma that could had occasioned meningitis. He developed headache, confusion, gait abnormalities and focal neurological deficits. Even though a polineuropathy was previously suspected and was treated with intravenous inmunoglobulin, we believe that the isolation of the germ in the CSF had a pathogenic role. After isolating the germ in the CSF we considered the possibility that it may have been the cause of the clinical symptoms shown by our patient and that its entryway was the head injury prior to his admission. P. carboxydivorans was the only microorganism isolated in the CSF. The bacterium was identified by molecular methods of rRNA 16S sequencing in the National Center of Microbiology (ISCIII, Madrid, Spain). Te patient was treated with trimethoprim-sulfamethoxazole according to the antibiogram and also because of the high level of this antibiotic in the CSF. The evolution was good but we could not verify the sterility of the CSF because the patient did not agree to a new lumbar puncture due to the fact that he was feeling well. To our knowledge, this is the first documented case of isolation of $P$. carboxydivorans in human cerebrospinal fluid.

\section{Conclusion}

This is the first documented case of isolating P. carboxydivorans in human CSF in a patient with a probable meningitis. Further research is needed in order to determine its pathogenic role in human infections.

\section{Abbreviations}

BAL: Bronchoalveolar lavage fluid; CRP: C-reactive protein; CSF: Cerebrospinal fluid; CT: Computed tomography; ECG: electrocardiogram; EMG: Electromyography; MN: Mononuclear; ND: No data; Neg: Negative; RA: Rheumatoid arthritis; SSIs: surgical site infections; TBI: Traumatic brain injury

\section{Acknowledgments}

Gabriela Harsulescu, Carlos De Cabo De La Vega and Alfonso Cuenca-Navarro for editorial assistance. We are indebted to the Hospital Research Committe from the Complejo Hospitalario Universitario de Albacete for fundig. We are particulary indebted to Dr. Juan Antonio Sáez Nieto of National Center of Microbiology (ISCIII, Madrid) for identification of P. carboxydivorans.

\section{Funding}

This work was funded by the Hospital Research Committee from the Complejo Hospitalario Universitario de Albacete Foundation.

Availability of data and materials

Not applicable.

Authors' contributions

AN, NC, CS, AE, JG, JAS, and JS made substantial contribution to the conception of the study, and analysis and interpretation of data. All authors read and approved the final manuscript.

Competing interests

The authors declare that they have no competing interests.

\section{Consent for publication}

Written informed consent was obtained from the patient for publication of this case report and any accompanying images. A copy of written consent is available for review by the Editor of this journal.

Ethics approval and consent to participate

Not applicable.

\section{Publisher's Note}

Springer Nature remains neutral with regard to jurisdictional claims in published maps and institutional affiliations.

\section{Author details}

${ }^{1}$ Internal Medicine Department, University General Hospital, C/ Hermanos Falcó s/n, 02006 Albacete, Spain. ${ }^{2}$ Microbiology Department, University General Hospital, C/Hermanos Falcó s/n, 02006 Albacete, Spain. ${ }^{3}$ National Center of Microbiology (ISCIII, Madrid), Madrid, Spain.

Received: 25 July 2016 Accepted: 9 June 2017

Published online: 06 July 2017

References

1. Henssen A. Beiträge zur Morphologie und Systematik der thermophilen Actinomyceten. Arch Mikrobiol. 1957;26:373-414

2. Park SW, Park ST, Lee JE, Kim YM. Pseudonocardia carboxydivorans sp. nov., a carbon monoxide-oxidizing actinomycete, and an emended description of the genus Pseudonocardia. Int J Syst Evol Microbiol. 2008;58:2475-8.

3. Li J, Zhao G-Z, Varma A, Qin S, Xiong Z, Huang H-Y, et al. Pseudonocardia xishanensis sp. nov., an endophytic actinomycete isolated from the roots of Artemisia annua L. Int J Syst Evol Microbiol. 2012;62:2395-9.

4. Mueller UG, Ishak H, Sen R, Gutell RR. Placement of attine ant-associated Pseudonocardia in a global Pseudonocardia phylogeny (Pseudonocardiaceae,Actinomycetales):a test of two symbiont-association models. Antonie Van Leeuwenhoek. 2010;98:185-212.

5. Sen $P$, et al. Generalized antifungal activity and 454 screening of Pseudonocardia and Amycolaptosis bacteria in nests of fungus-growing ants. Proc Natl Acad ScIU S A. 2009;106:805-10.

6. Scher JU, et al. The lung microbiota in early rheumatoid arthritis and autoinmmunity. Microbiome. 2016;4:60.

7. Drancourt M, Bollet C, Carlioz A, MartelinR GJP, Raoult D. 16S ribosomal DNA sequence analysis of a large collection of environtmental and clinical unidentifiable bacterial isolates. J Clin Microbiol. 2000;38:3623-530.

8. Kourberti IS, Vakis AF, Papadakis JA, Karabetsos DA, Bertsias G, Filippou M, et al. Infections in traumatic brain injury patients. Clin Microbiol Infect. 2012;18:359-64 